\title{
Defining The Learning Curve of Single-Port Inflatable Mediastinoscopy With Simultaneous Laparoscopic-Assisted Surgery For Radical Esophagectomy
}

\section{Jinpeng Shi}

Fifth Affiliated Hospital of Sun Yat-sen University

\section{Xiaojian Li}

Fifth Affiliated Hospital of Sun Yat-sen University

Tianchi Wu

Fifth Affiliated Hospital of Sun Yat-sen University

\section{Xiangwen Wu}

Fifth Affiliated Hospital of Sun Yat-sen University

\section{Xiaojin Wang}

Fifth Affiliated Hospital of Sun Yat-sen University

\section{Hua Cheng}

Fifth Affiliated Hospital of Sun Yat-sen University

\section{Hongcheng Zhong}

Fifth Affiliated Hospital of Sun Yat-sen University

\section{Bin Zhang}

Fifth Affiliated Hospital of Sun Yat-sen University

\section{Beilong Zhong}

Fifth Affiliated Hospital of Sun Yat-sen University

\section{Xiangfeng Gan}

Fifth Affiliated Hospital of Sun Yat-sen University

\section{Qingdong Cao ( $\nabla$ shijp21@163.com )}

The Fifth Affiliated Hospital of Sun Yat-sen University

\section{Research article}

Keywords: Esophageal cancer, esophagectomy, endoscope, mediastinum, minimally invasive esophagectomy

Posted Date: July 20th, 2021 
DOI: https://doi.org/10.21203/rs.3.rs-643095/v1

License: (c) (1) This work is licensed under a Creative Commons Attribution 4.0 International License. Read Full License 


\section{Abstract}

Background Single-port inflatable mediastinoscopy with simultaneous laparoscopic-assisted surgery for radical esophagectomy is a promising surgical method with high technical requirements and needs team cooperation. Therefore, it is necessary to define a learning curve to guide personnel training and improve the safety of these surgical techniques.

Method This study prospectively analyzed the data of 79 consecutive patients, who underwent the surgery in the Fifth Affiliated Hospital of Sun Yat-sen University from October 2016 to May 2018. All of these patients were treated by the same surgical team with extensive experience in thoracotomy, laparotomy, thoracoscopic surgery and laparoscopic surgery. The learning curve was analyzed by cumulative summation (CUSUM) analysis, with the assessment of operative time, estimated blood loss, and postoperative complications.

Result By analyzing these data, The scatter diagram of every measure showing a declining situation. The learning curve decreased beginning at $25^{\text {th }}$ operation. All patients were chronologically divided into two groups, the group 1 (the first 25 patients) and the group 2 (the last 54 patients). The median estimated blood loss of group 2 was lower than group $1(200 \mathrm{vs} 100 \mathrm{ml}, p<0.05)$. No other clinic or pathologic characteristics were observed as significantly different.

Conclusion For a surgical team with extensive experience in thoracotomy, laparotomy, thoracoscopic surgery and laparoscopic surgery, 25 cases are needed before becoming proficient in this surgery.

\section{Background}

Esophageal cancer is one of the leading causes of cancer death worldwide[2]. There are a large number of cases of esophageal cancer in China[2, 6]. At present, the basic treatment strategy is a combination of surgery, radiotherapy and chemotherapy[11]. Although the incidence of postoperative complications and mortality after esophagectomy is relatively high, radical surgery is still the basic treatment for early and local late esophageal cancer[11, 12]. In recent years, with the continuous development of minimally invasive and endoscopic surgery, the postoperative mortality of esophageal cancer has been greatly reduced due to the benefits and advantages of minimally invasive surgery, including less intraoperative blood loss, rapid postoperative recovery and fewer postoperative complications[1, 5, 13]. Increasing numbers of minimally invasive methods are used for esophageal cancer surgery. Thoracoscopy combined with laparoscopic radical esophagectomy is a common operation which could increase the risk of many perioperative cardiopulmonary complications, often affecting the prognosis of patients[4]. Robot-assisted radical esophagectomy is a technically demanding, costly and time-consuming operation. To reduce the incidence of postoperative cardiopulmonary complications of radical esophagectomy, during 2015 and 2016, Professor Fujiwara developed inflatable mediastinoscopy-assisted transhiatal radical esophagectomy[7]. To reduce trauma and avoid a double incision in the neck, on the basis of Professor Fujiwara's surgical method, we propose a surgical approach to the bilateral RLN lymph nodes 
with a single left-neck incision and further improved the surgical method to single-port inflatable mediastinoscopy with simultaneous laparoscopic-assisted surgery for radical esophagectomy[10, 14].

It takes time and experience to fully master the skills of single-port inflatable mediastinoscopy with simultaneous laparoscopic-assisted surgery for radical esophagectomy, even for surgical teams with extensive experience in thoracotomy, laparotomy, thoracoscopic surgery and laparoscopic surgery. Therefore, it is necessary to define the learning curve of single-port inflatable mediastinoscopy with simultaneous laparoscopic-assisted surgery for radical esophagectomy. We used cumulative summation analysis (CUSUM) [15] to define the learning curve of this surgical method to guide the training process for surgical personnel and improve the safety of these surgical techniques.

\section{Patients And Methods}

All 79 patients included in this study were treated by the same surgical team using single-port inflatable mediastinoscopy with simultaneous laparoscopic-assisted surgery for radical esophagectomy in the Fifth Affiliated Hospital of Sun Yat-sen University, From October 2016 to May 2018. The surgeon was always the chief physician, and the first assistant was one of two relatively fixed doctors (deputy chief physician and senior attending physician). All three have extensive experience in thoracotomy, laparotomy, thoracoscopic surgery and laparoscopic surgery.

The surgical team started our first single-port inflatable mediastinoscopy with simultaneous laparoscopic-assisted surgery for radical esophagectomy after we propose a surgical approach to the bilateral RLN lymph nodes with a single left-neck incision and further improved the surgical method to single-port inflatable mediastinoscopy with simultaneous laparoscopic-assisted surgery for radical esophagectomy $[10,14]$ on the basis of Professor Fujiwara's surgical method.

\section{Patients}

The inclusion criteria in our study were the same as those for open operations or traditional transthoracic or thoracotomy, including some challenging cases. Inclusion criteria were: (1) preoperative endoscopic and biopsy specimens pathologically diagnosed clinical stage I-III esophageal cancer; (2) physical condition of the patient suitable for radical resection; (3) tumor size within the resectable range based on contrast computed tomography of the chest and abdomen and PET-CT, regardless of the use of preoperative neoadjuvant therapy; and (4) no other tumors. The exclusion criteria were as follows:(1) severe complications that did not allow for surgery; (2) preoperative tumor stage evaluated to be higher than stage IVA; and (3) refusal to surgical treatment. This study was approved by the institutional review board of our hospital. Written informed consent was obtained from the patient.

We used the cross-point of the cumulative sum (CUSUM) plots to assign the first 25 patients who underwent single-port inflatable mediastinoscopy with simultaneous laparoscopic-assisted surgery for radical esophagectomy to group 1 (the first 25 patients) and the remaining 54 patients to group 2 (the last 54 patients). Retrospectively collected data included surgical time (from the beginning of skin 
incision to the end of suture incision), estimated blood loss, the use of preoperative neoadjuvant therapy, number of retrieved lymph nodes, postoperative complications, and postoperative hospital length of stay.

A learning curve was considered complete when a point for decreasing surgical time was observed from the CUSUM plot.

\section{Surgical Technique}

Single-port inflatable mediastinoscopy with simultaneous laparoscopic-assisted surgery for radical esophagectomy mainly includes left neck surgery and abdominal surgery[10, 14].

The operation was performed by two operation teams. One team made the cervical incision and performed the single-port mediastinoscopy for the dissection of upper mediastinal lymph nodes and upper esophageal. Simultaneously, another team performed laparoscopy for the dissection of lower mediastinal lymph nodes and lower esophageal segment. (Fig. 1) Carbon dioxide was simultaneously pumped into the mediastinum and abdominal cavity to maintain the pressure at about $10 \sim 12 \mathrm{mmHg}$. The novel inflatable mediastinoscopy ensured an excellent surgical view in the mediastinal and abdominal operative field, markedly elevating the surgical safety and reducing the difficulty for the resection of the whole mediastinal lymph nodes and dissection of the whole esophagus. Thus, this surgical method overcomes the defects of the conventional non-transthoracic esophagectomy. In addition, the clear surgical view can effectively reduce the operation duration and intraoperative blood loss as compared with the thoracic-abdominal endoscopic or conventional open transthoracic esophagectomy of esophageal cancer radical surgery. Moreover, the total esophageal excision and lymph nodes dissection can be appropriately performed.

Statistical Analysis

Data were collected retrospectively by the same doctor. They were reported as mean \pm SD or medians (ranges) for continuous variables and as frequency (\%) for categoric variables. SPSS 25.0 software (IBM, Armonk, NY) was used for the data analysis. A two-sided $p$ value of less than 0.05 was considered to indicate a significant difference.

We used the CUSUM plots for analyzing learning curve for Single-port inflatable mediastinoscopy with simultaneous laparoscopic-assisted surgery for radical esophagectomy in patients with esophageal cancer. The CUSUM method is valuable in the early detection of trends in data[8]. First, we selected the average value of all our data points as the reference value. Then, this reference value was subtracted from each data point in succession, and any remainder was added to the previous sum. For instance, the CUSUM for the first patient was the difference between the value for the first patient and the mean for all patients. The CUSUM for the second patient was the previous patient's CUSUM added to the difference obtained for the second patient. We calculated each CUSUM in chronologic order and analyzed the surgical time data by visually inspecting the plots. A learning curve was considered complete when a point for decreasing surgical time was observed from the CUSUM plot. 


\section{Results}

A total of 79 patients were included, including 54 males and 25 females, with an age of $61.49 \pm 9.28$ years (42-84). All patients underwent single-port inflatable mediastinoscopy with simultaneous laparoscopic-assisted surgery for radical esophagectomy, and there was no conversion to other surgical methods during the operation.

By visually inspecting the CUSUM plots, we saw that a decreasing trend for total surgical time began at 25th operation (Fig. 2). Patients were divided into two groups according to chronologic order: group 1 comprised the first 25 esophagectomy cases, and group 2 comprised the next 54 cases. Patients in the two groups were comparable in age, sex, preoperative comorbidities, and histology (Table 1).

Table 1. Baseline characteristics of patients undergoing single-port inflatable mediastinoscopy with simultaneous laparoscopic-assisted surgery for radical esophagectomy.

\begin{tabular}{|c|c|c|c|}
\hline Variable & Group $1 \rrbracket n=25 \rrbracket$ & Group 2】n=54】 & $\mathrm{p}$ \\
\hline Age $(y \rrbracket$ & $59.72 \pm 8.98$ & $62.31 \pm 9.38$ & 0.38 \\
\hline Sex & & & 0.001 \\
\hline Male & $20 \rrbracket 80.0 \rrbracket$ & $34 \llbracket 63.0 \rrbracket$ & \\
\hline Female & $5 \llbracket 20.0 \rrbracket$ & $20 \rrbracket 37.0 \rrbracket$ & \\
\hline \multicolumn{4}{|l|}{ Comorbidities } \\
\hline Chronic obstructive pulmonary disease & $1 \rrbracket 4.0 \rrbracket$ & $3 \rrbracket 5.6 \rrbracket$ & 0.56 \\
\hline Hypertension & $7 \rrbracket 28.0 \rrbracket$ & $11 \rrbracket 20.4 \rrbracket$ & 0.16 \\
\hline Diabetes & $2 \varangle 8.0 \bigotimes$ & $4 \rrbracket 7.4 \rrbracket$ & 0.86 \\
\hline Cerebral infarction & $2 \varangle 8.0 \bigotimes$ & $0 \rrbracket 0 \otimes$ & 0.00 \\
\hline Hepatic cirrhosis & $0 \otimes 0 \bigotimes$ & 1ه1.9凶 & 0.17 \\
\hline Atrial fibrillation & $0 \otimes 0 \rrbracket$ & 1区1.9凶 & 0.17 \\
\hline Coronary artery disease & $0 \otimes 0 \rrbracket$ & $5 \llbracket 9.3 \rrbracket$ & 0.001 \\
\hline \multicolumn{4}{|l|}{ Histology } \\
\hline Squamous & $24 \rrbracket 96.0 \rrbracket$ & $51 \otimes 94.4 \rrbracket$ & 0.561 \\
\hline Adenocarcinoma & $1 \rrbracket 4 \rrbracket$ & $0 \otimes 0 \rrbracket$ & 0.003 \\
\hline Neuroendocrine carcinoma & 0ख0囚 & $3 \rrbracket 5.6 \rrbracket$ & 0.013 \\
\hline
\end{tabular}

Data are presented as mean \pm SD or number (\%). 
The median total surgical time and intraoperative variables and postoperative short-term outcomes were summarized are reported in Table 2. Comparing groups 1 and 2, the median total surgical time was 300 vs 247.5 minutes. By drawing the scatter diagram of the operative time (as shown in Fig. 3), we knew that the overall trend is as follows: as the number of operation cases gradually increases, the operative time gradually shortens. The median estimated blood loss was $200 \mathrm{vs} 100 \mathrm{ml}$. The estimated blood loss as well showed a descending trend (as shown in Fig. 4). The median number of lymph nodes harvested was $15.9 \pm 6.5$ in group 1 and $18.1 \pm 7.3$ in group 2 , which revealed group 2 retrieved more lymph nodes. Pathologic staging was not different between the two groups. Intensive care unit stay, postoperative hospital length of stay, and the incidence of postoperative complication in group 2 was lower than that in group 1. postoperative complication contains (1) postoperative anastomotic leakage, (2) pulmonary infection, (3) pleural effusion requiring treatment, (4) wound infection, (5) chylothorax, (6) reflux esophagitis, (7) vocal cord palsy.

Table 2. Intraoperative and Postoperative Variables 


\begin{tabular}{|c|c|c|c|}
\hline Variablea & $\begin{array}{l}\text { Group } 1 \\
(n=25)\end{array}$ & $\begin{array}{l}\text { Group } 2 \\
(n=54)\end{array}$ & $\mathrm{p}$ \\
\hline Surgical time & $300 \rrbracket 217 \sim 470 \rrbracket$ & $247.5 \rrbracket 160 \sim 301 \rrbracket$ & 0.10 \\
\hline Estimated blood loss, ml & $200 \rrbracket 20 \sim 500 \rrbracket$ & 100(30 200) & 0.00 \\
\hline Retrieved lymph nodes, No. & $15.9 \pm 6.5$ & $18.1 \pm 7.3$ & 0.89 \\
\hline Postoperative Hospital Length of stay, $d$ & $22.7 \pm 26.0$ & $20.5 \pm 11.7$ & 0.06 \\
\hline Intensive care unit & $3 \otimes 12.0 \otimes$ & $7 \rrbracket 13.0 \rrbracket$ & 0.81 \\
\hline \multicolumn{4}{|l|}{ Postoperative complication } \\
\hline Anastomotic leakage & $5 \rrbracket 20.0 \rrbracket$ & $5 \otimes 9.3 \rrbracket$ & 0.46 \\
\hline Pulmonary infection & $2(8.0)$ & $7(13.0)$ & 0.19 \\
\hline Hoarseness after 3 months & $4(16.0)$ & $7(13.0)$ & 0.48 \\
\hline Wound infection & $5 \rrbracket 20.0 \rrbracket$ & $9 \otimes 13.0 \rrbracket$ & 0.35 \\
\hline Pleural effusion requiring treatment & $8 \llbracket 32.0 \rrbracket$ & $13(24.1)$ & 0.17 \\
\hline Chylothorax & $1(4.0)$ & $1(1.9)$ & 0.27 \\
\hline Reflux esophagitis & $1(4.0)$ & $1(1.9)$ & 0.27 \\
\hline \multicolumn{4}{|l|}{ Tumor location } \\
\hline Upper & $5(20.0)$ & $6(11.1)$ & 0.043 \\
\hline Middle & $6(24.0)$ & 19(35.2) & 0.033 \\
\hline Lower & $14(56.0)$ & $29(53.7)$ & 0.689 \\
\hline Neoadjuvant & $5 \rrbracket 20.0 \rrbracket$ & $11 \rrbracket 20.4 \rrbracket$ & 0.94 \\
\hline \multicolumn{4}{|l|}{ Pathologic stage } \\
\hline 0 & 0 & $2(3.7)$ & 0.048 \\
\hline IA & 0 & $1(1.9)$ & 0.171 \\
\hline IB & $9(36.0)$ & $12(22.2)$ & 0.025 \\
\hline IIA & $4(16.0)$ & $8(14.8)$ & 0.789 \\
\hline IIB & $4(16.0)$ & $4(7.4)$ & 0.023 \\
\hline IIIA & $2(8.0)$ & $5(9.3)$ & 0.717 \\
\hline IIIB & $6(24.0)$ & $22(40.0)$ & 0.001 \\
\hline
\end{tabular}

a Continuous data are presented as mean \pm SD or median (range) and categoric data as number (\%). 


\section{Discussion}

Single-port inflatable mediastinoscopy with simultaneous laparoscopic-assisted surgery for radical esophagectomy was performed by two groups of doctors from the same surgical team. One group underwent neck surgery with single-port inflatable mediastinoscopy to dissect the upper esophagus and dissection the cervical lymph nodes, while the other group dissected the lower esophagus and dissected the lower mediastinal lymph nodes by laparoscopy. $\mathrm{CO}_{2}$ was continuously filled into the mediastinum and abdominal cavity under a pressure of $10 \mathrm{mmHg}$ to ensure a good surgical field of vision in the mediastinal and abdominal surgery area and easy operation. This method not only minimizes the surgical incision but also overcomes the shortcomings of traditional thoracotomy and effectively reduces the operation time and the amount of intraoperative blood loss. Compared with transthoracic surgery, nontransthoracic surgery can effectively relieve postoperative pain and postoperative cardiopulmonary complications and expand the indications of radical resection of the esophagus[3, 8, 9].

Currently, minimally invasive surgery is developing rapidly. For doctors with extensive experience in minimally invasive laparoscopic and video-assisted thoracoscopic surgery, it takes a certain amount of time and experience to master a new operation skillfully. Especially for single-port endoscope surgery, the operating instruments are parallel to the endoscope, and the operations are also performed simultaneously, which increases the difficulty of the operation and the skills required of the operator. Therefore, it is necessary to define the learning curve of single-port inflatable mediastinoscopy simultaneous laparoscopic-assisted surgery for radical esophagectomy. The learning curve represents a process of gradually completing and skillfully mastering a certain technology. Generally, the learning curve has two stages, a rapid rising period and a plateau period. When the curve reaches the plateau, it shows that the technical operation has been skillfully completed and is stable, that is, the learning curve has been overcome. The CUSUM, which can help to discern the trend of a data set intuitively, was proved particularly valuable when the learning curve was analyzed. In the presented series, the CUSUM plots of overall surgical time presented inflection points at the 25th case, implying that 25 operations were needed to gain early proficiency in Single-port inflatable mediastinoscopy with simultaneous laparoscopicassisted surgery for radical esophagectomy for an operative team that is familiar with standard thoracic surgical procedures. No studies until now have reported the learning curve with Single-port inflatable mediastinoscopy with simultaneous laparoscopic-assisted surgery for radical esophagectomy.

The reason why this study chose operative time, the amount of intraoperative blood loss and the incidence of postoperative complications as the observation values, is because a reduction in operative time can indicate that the operator was more skillful. The application of three observation indicators can further show that on the basis of a skilled operation, the safety of the operation also improved. However, the median number of lymph nodes harvested in group 2 was greater than in group 1, which suggested that the early oncologic results of the patients may be relative to the surgeon's experience. In order to achieve a relatively lower incidence of adverse events and shorter surgical time, some surgeons prefer to avoid challenging cases at the beginning of a new surgical procedure, which may result in selection bias; but selection bias might also influence the learning curve analysis. In our series, the selection criteria for 
Single-port inflatable mediastinoscopy with simultaneous laparoscopic-assisted surgery for radical esophagectomy were identical to open or conventional MIE, which may present more reliable evidence to guide novice surgeon training and staff in centers implementing this new technique.

This study is a retrospective analysis, and all patients strictly aligned with the surgical indications. However, there are some limitations in this study, such as underwent intraoperative exploration for the existence of thoracic adhesions and other factors that may affect the difficulty of the operation. Hoarseness may be caused by intraoperative laryngeal nerve injury, some patients gradually recover after a few days was treated with intravenous infusion of ganglioside gradually recovered within 1 to 2 months because the nerve injury was temporary and reversible. Only few patients still had hoarseness. It was difficult to determine whether a recurrent laryngeal nerve injury was present.

We focused on surgical time for analysis because operation time is the most widely used marker for the learning curve in previous reports. Postoperative morbidity, death, management of chest tubes and nasogastric drainage, and initiation of oral intake were also important indicators of a learning curve. These need further obsearvation.

\section{Abbreviations}

$\mathrm{RLN}$ : recurrent laryngeal nerve

PET/CT: positron emission tomography / computedtomography

\section{Declarations}

Ethics approval and consent to participate[The hospital ethics committee approval was granted of this article. Consent for publication was obtained from the paitent.

Consent for publication: Written informed consent was obtained from the patients for publication of this article.

Availability of data and materials[The datasets used and/or analysed during the current study are available from the corresponding author on reasonable request.

Competing interests: There were no conflicts of interest.

Acknowledgements: The authors are grateful to all the surgeons in the Cardiothoracic surgery for providing the data.

Funding: This work was supported by National Key Research and Development Program of China (2018YFC0910600). 
Authors' contributions: Qingdong Cao and Xiangfeng Gan designed and supervised the work. Jinpeng Shi and Xiaojian Li analyzed and interpreted the data. All the other authors contributed equally to the data collection and writing.

\section{References}

1. Biere S S, van Berge Henegouwen M I, Maas K W, Bonavina L, Rosman C, Garcia J R, et al. Minimally invasive versus open oesophagectomy for patients with oesophageal cancer: a multicentre, open-label, randomised controlled trial. Lancet. 2012; 379(9829), 1887-1892.

2. Bray F, Ferlay J, Soerjomataram I, Siegel R L, Torre L A, Jemal A. Global cancer statistics 2018: GLOBOCAN estimates of incidence and mortality worldwide for 36 cancers in 185 countries. CA Cancer $\mathrm{J}$ Clin. 2018; 68(6), 394-424.

3. Bumm R, Hölscher A H, Feussner H, Tachibana M, Bartels H, Siewert J R. Endodissection of the thoracic esophagus. Technique and clinical results in transhiatal esophagectomy. Ann Surg. 1993; 218(1), 97-104.

4. Canet J, Gallart L, Gomar C, Paluzie G, Vallès J, Castillo J, et al. Prediction of postoperative pulmonary complications in a population-based surgical cohort. Anesthesiology. 2010; 113(6), 1338-1350.

5. Feng $M X$, Wang $H$, Zhang $Y$, Tan $L$ J, Xu Z L, Qun W. Minimally invasive esophagectomy for esophageal squamous cell carcinoma: a case-control study of thoracoscope versus mediastinoscope assistance. Surg Endosc. 2012; 26(6), 1573-1578.

6. Feng R M, Zong Y N, Cao S M, Xu R H. Current cancer situation in China: good or bad news from the 2018 Global Cancer Statistics? Cancer Commun (Lond). 2019; 39(1), 22.

7. Fujiwara H, Shiozaki A, Konishi H, Kosuga T, Komatsu S, Ichikawa D, et al. Single-Port Mediastinoscopic Lymphadenectomy Along the Left Recurrent Laryngeal Nerve. Ann Thorac Surg. 2015; 100(3), 1115-1117.

8. Fujiwara H, Shiozaki A, Konishi H, Kosuga T, Komatsu S, Ichikawa D, et al. Perioperative outcomes of single-port mediastinoscope-assisted transhiatal esophagectomy for thoracic esophageal cancer. Dis Esophagus. 2017; 30(10), 1-8.

9. Fujiwara H, Shiozaki A, Konishi H, Otsuji E. Transmediastinal approach for esophageal cancer: A new trend toward radical surgery. Asian J Endosc Surg. 2019; 12(1), 30-36.

10. Gan X, Wang X, Zhang B, Cheng H, Zhong B, Zhong H, et al. Lymphadenectomy Along Bilateral Recurrent Laryngeal Nerves Under Single-Incision Mediastinoscopy. Ann Thorac Surg. 2020; 109(6), e449e452. 
11. Napier K J, Scheerer M, Misra S. Esophageal cancer: A Review of epidemiology, pathogenesis, staging workup and treatment modalities. World J Gastrointest Oncol. 2014; 6(5), 112-120.

12. Raymond D P, Seder C W, Wright C D, Magee M J, Kosinski A S, Cassivi S D, et al. Predictors of Major Morbidity or Mortality After Resection for Esophageal Cancer: A Society of Thoracic Surgeons General Thoracic Surgery Database Risk Adjustment Model. Ann Thorac Surg. 2016; 102(1), 207-214.

13. Takeuchi H, Miyata H, Ozawa S, Udagawa H, Osugi H, Matsubara $\mathrm{H}$, et al. Comparison of Short-Term Outcomes Between Open and Minimally Invasive Esophagectomy for Esophageal Cancer Using a Nationwide Database in Japan. Ann Surg Oncol. 2017; 24(7), 1821-1827.

14. Wang X, Li X, Cheng H, Zhang B, Zhong H, Wang R, et al. Single-Port Inflatable Mediastinoscopy Combined With Laparoscopic-Assisted Small Incision Surgery for Radical Esophagectomy Is an Effective and Safe Treatment for Esophageal Cancer. J Gastrointest Surg. 2019; 23(8), 1533-1540.

15. Wohl H. The cusum plot: its utility in the analysis of clinical data. N Engl J Med. 1977; 296(18), 10441045.

\section{Figures}




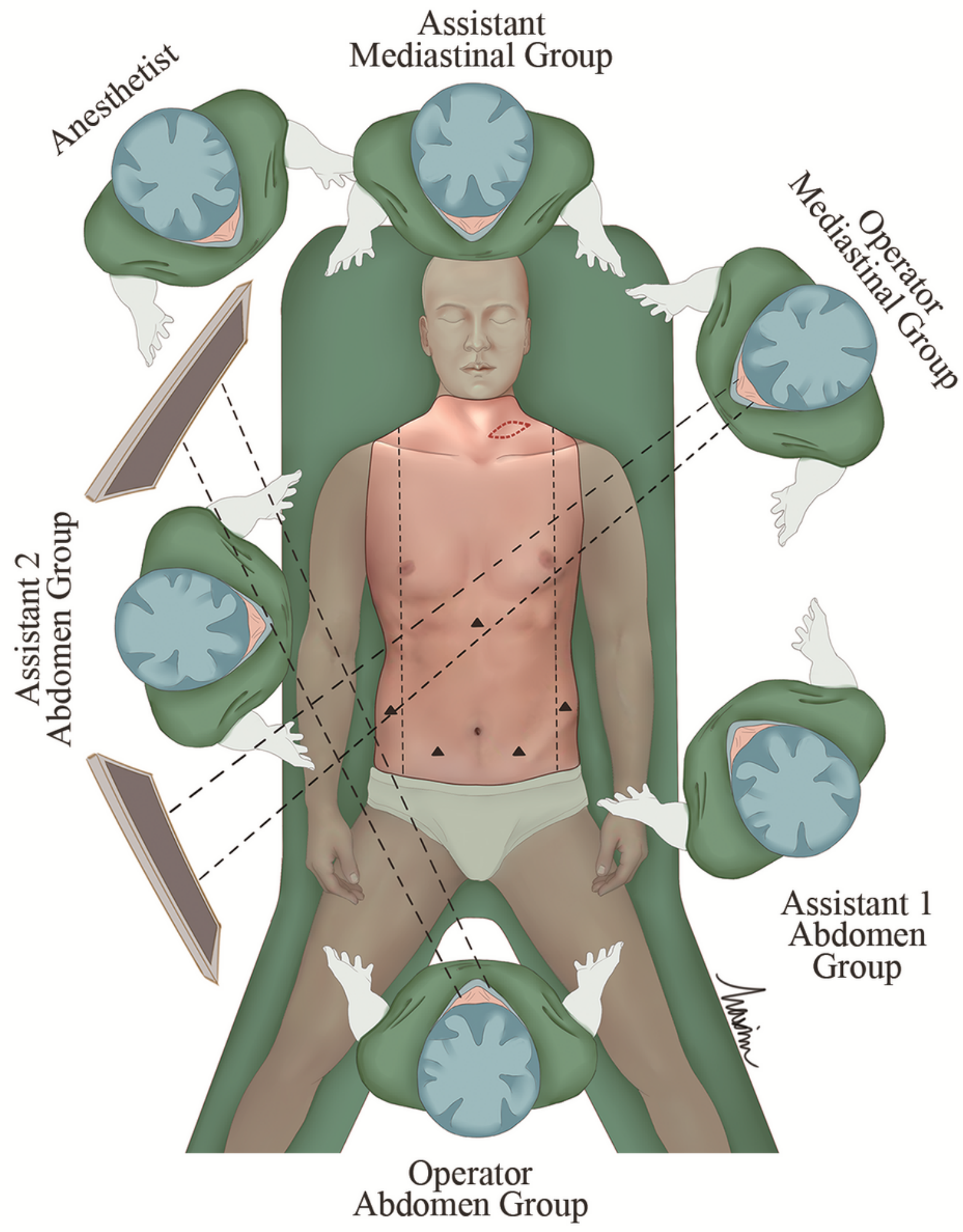

Figure 1

Position of the operators, assistants, and the anesthetist during the surgery. 


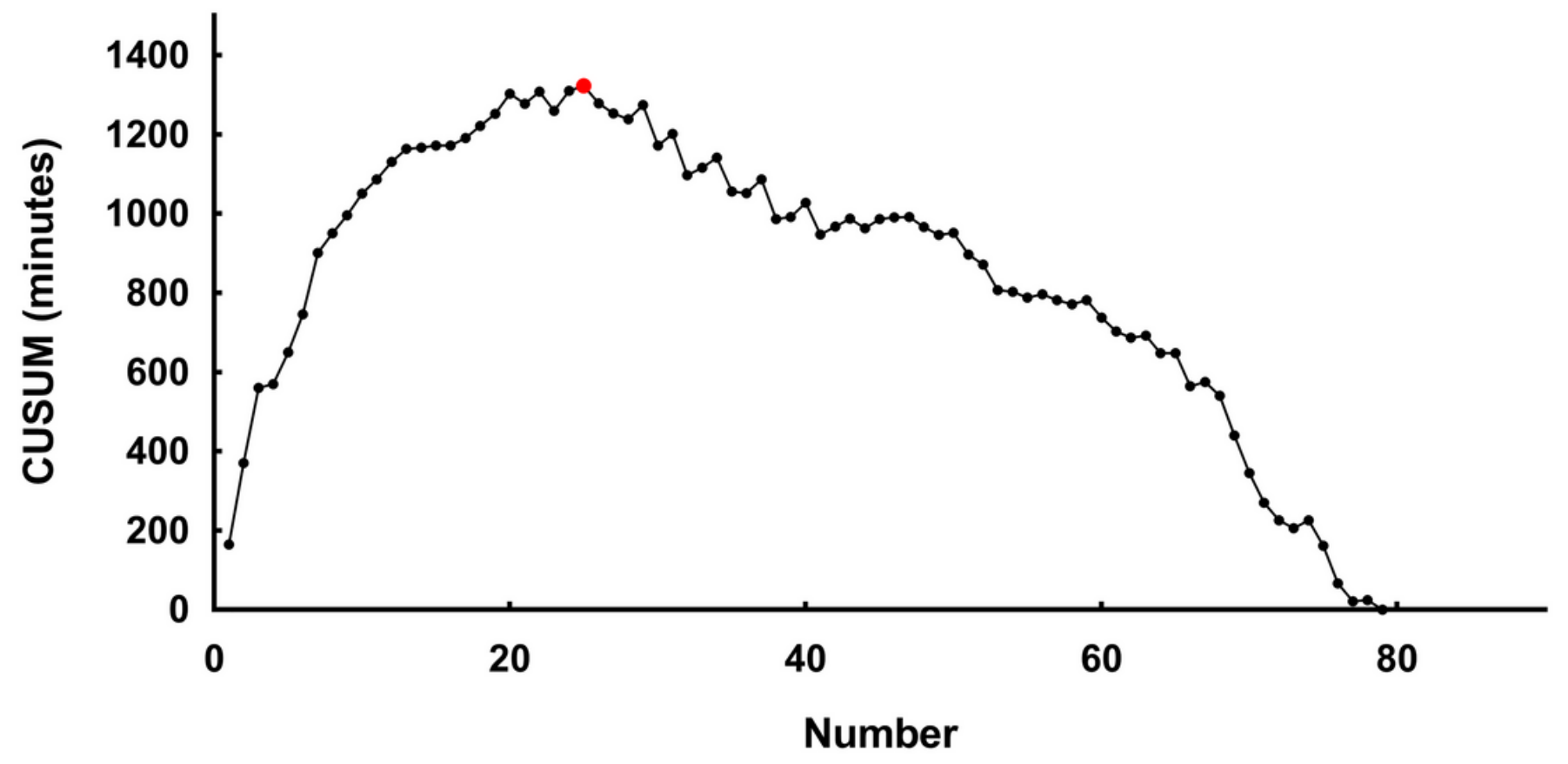

Figure 2

A visual inspection of the cumulative sum (CUSUM) plots shows a decrease for total surgical time begins at the 25th operation.

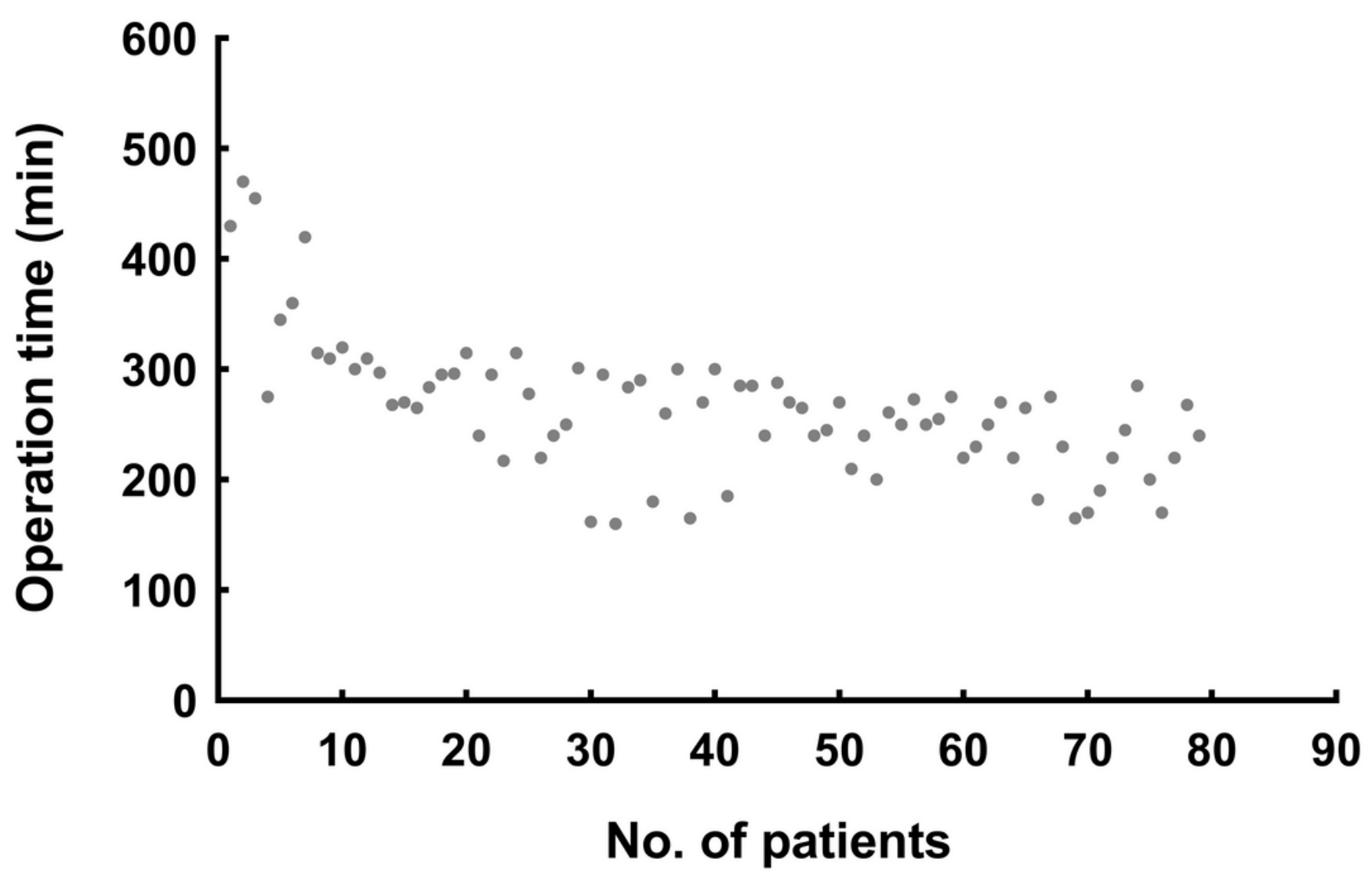


Figure 3

The scatter diagram of the operative time shows that with the implementation of a new surgical technique, the operative time was longer at the beginning, and gradually shortened with the increase of the number of surgery cases, showing a declining situation, and finally reached a plateau.

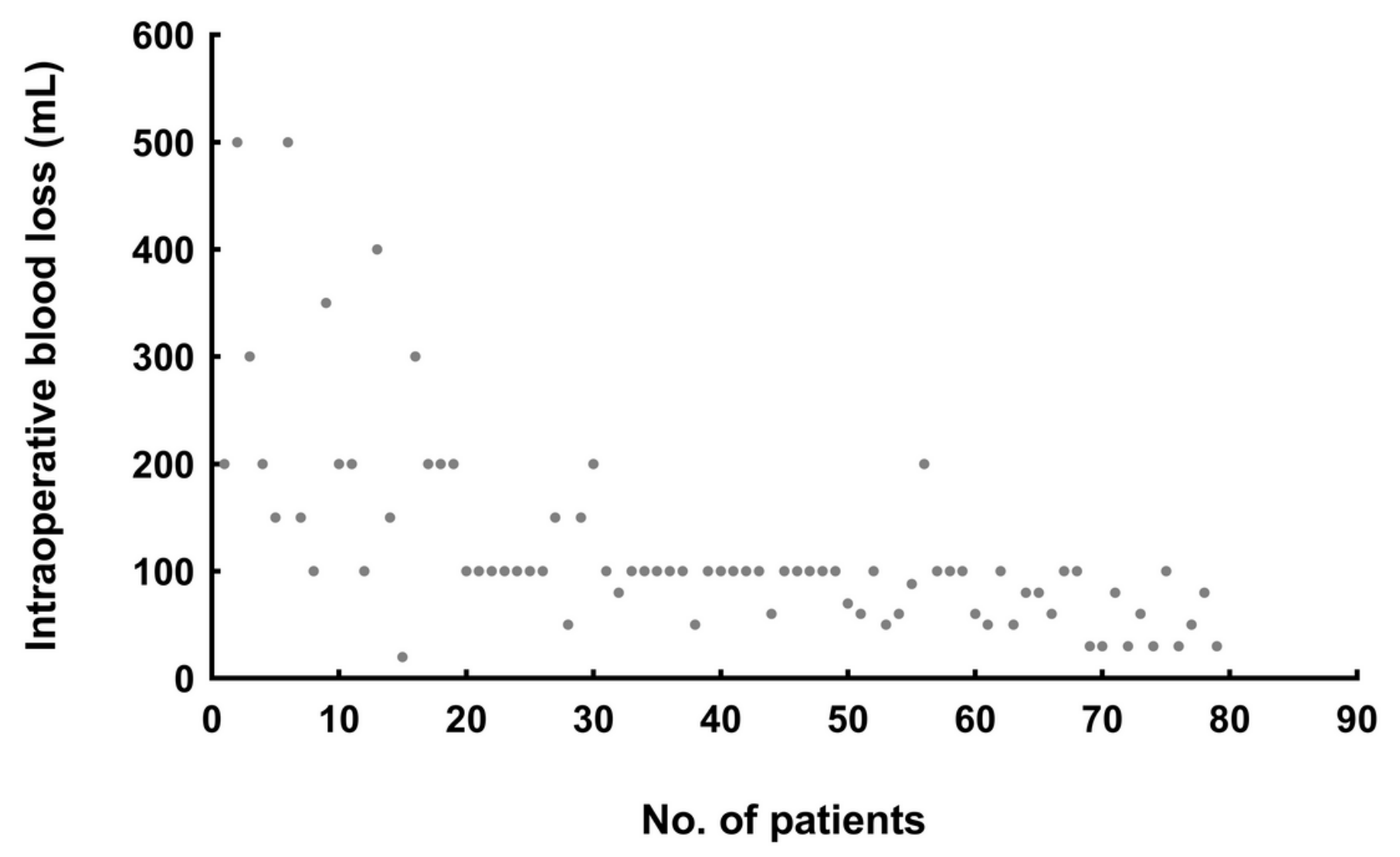

Figure 4

The scatter diagram of the amount of intraoperative blood loss shows that with the implementation of a new surgical technique, the amount of intraoperative blood loss is large, with the increase of the number of surgery cases, the operation gradually skilled, the amount of intraoperative blood loss gradually decreased, showing a declining situation, and finally reached a plateau. 